\title{
New Vector Ordering in the RedGreenBlue Colour Model with Application to Morphological Image Magnification *
}

\author{
Valérie DE WITTE and Stefan SCHULTE and Etienne E. KERRE \\ Department of Applied Mathematics and Computer Science \\ Fuzziness and Uncertainty Modelling Research Unit \\ Ghent University, Krijgslaan 281 (Building S9) \\ B-9000 Gent, Belgium ${ }^{\dagger}$ \\ E-mail:Valerie.DeWitte@gmail.com
}

Received: 31-07-2007

Revised: 26-09-2007

\begin{abstract}
In this paper we present a new vector ordering $\leq_{R G B}$ for colours modelled in the RedGreenBlue colour model. The RedGreenBlue colour model becomes with this new ordering and associated minimum and maximum operators a complete lattice. We also have defined a complement co for colours in the RedGreenBlue model, with which our new ordering is compatible. As an application we illustrate the extension of our morphological magnification method, described in ${ }^{8}$ and ${ }^{1}$, towards colour images with sharp edges modelled in the RedGreenBlue model. There we have needed the compatibility of $\leq_{R G B}$ with co to detect the corners in an image using the hit-or-miss transformation. Experimental results demonstrate that our method gives very good results.
\end{abstract}

\section{Introduction}

Mathematical morphology has many applications in image processing such as pattern recognition, image segmentation, and image magnification. The application of morphological operators to colour images is not straightforward because of the need of the concept of a supremum and infimum operator, and thus, of an ordering in the used colour model. Some colour orderings in the RGB colour model have already been proposed in the literature. In this work we introduce a new approach for the ordering of colours in RGB. In section we explain our idea and we define the new colour ordering $\leq_{R G B}$. With this ordering new minimum and maximum are associated. We then define a complement $c o$ for colours in RGB and show that $\leq_{R G B}$ is compatible with co. Finally, in section, we have extended our morphological interpolation method, presented in ${ }^{8}$ and ${ }^{1}$, to magnify colour images in RGB with sharp edges.

\section{The RGB Colour Model}

A colour in the RGB colour model is obtained by adding the three colours red, green and blue in different combinations. Every colour in RGB is totally determined by its three colour components Red, Green and Blue. Therefore a colour can be defined as a vector in a three-dimensional space that can be represented as a unit cube using a Cartesian coordinate scheme, see figure 1. Every point in the cube represents then a vector (colour). The greyscale spectrum is characterised by the line between the black top Black with coordinates $(0,0,0)$ and the white top White $(1,1,1)$.

\footnotetext{
*New Vector Ordering in RGB with Morphological Image Magnification Application.

†Valérie DE WITTE, Valerie.DeWitte@gmail.com, BELGIUM.
} 


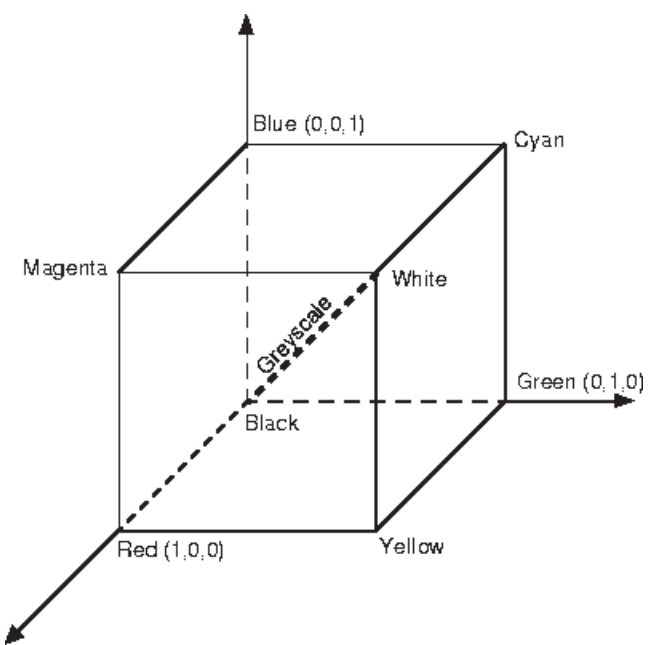

Fig. 1. A representation of the RGB colour model expressed in Cartesian coordinates.

Remark that in practice only a finite number of colours can be obtained in a colour model. Since each colour component is usually stored as 8 bits (one byte), i.e., the values of each colour component range in the interval $\left[0,2^{8}-1\right]$, a colour in a three-dimensional colour model is stored as a 24-bit colour. The values of each colour component by storage usually range in the interval $[0,255]$, but we can always scale them to the interval $[0,1]$. So we always work with finite colour models.

\section{A State-of-the-Art Overview}

Because there is no unambiguous way to order two or more colours, there exist different techniques to order colour. The most common used techniques are the componentwise ordering, also called marginal ordering, reduced ordering, partial ordering and conditional ordering, also called lexicographical ordering.

In marginal ordering each colour component is ordered independently and the operations are applied to each colour component of the image. But this approach does not exploit the correlation between the different colour components and because of this new colours can be introduced in an image.

In reduced ordering a single value is given to each multivariate value. So to each colour (vector) $c_{i}$ in the considered colour model is a scalar value $d_{i}=d\left(c_{i}\right)$, normally $d: \mathbb{R}^{3} \rightarrow \mathbb{R}$, added. After $d_{i}$ has been obtained for each $i$, the vectors $c_{1}, \ldots, c_{n}$ are ordered based on $d_{1}, \ldots, d_{n}$ as follows:

$$
c_{(1)} \leq \ldots \leq c_{(n)},
$$

where $c_{(r)}$ is the vector corresponding with the scalar value $d_{(r)}$, the $r$ th smallest element of the set $\left\{d_{1}, d_{2}, \ldots, d_{n}\right\}$. The output vector at each point in the image is, by definition of this ordering, one of the vectors in the original image so there is no possibility of introducing new colour vectors into the image. Usually some type of distance metric is used to perform reduced ordering. The output will of course depend on the used scalar-valued function, where characteristics of the human visual system, such as luminance, can be used as metric.

In partial ordering the colour vectors are partitioned into groups, which are then ordered.

In conditional ordering the colours are ordered using one component initially. In case multiple colours have the same initial component values, a second component is used to order the colours, and so on. Let $c=\left\{c_{1}, c_{2}, \ldots, c_{n}\right\}$ and $c^{\prime}=\left\{c_{1}^{\prime}, c_{2}^{\prime}, \ldots, c_{n}^{\prime}\right\}$ be two colour vectors in the considered colour model $(n \in \mathbb{N})$. An example of a lexicographical order can be

$$
c<c^{\prime} \quad \text { if }\left\{\begin{array}{l}
c_{1}<c_{1}^{\prime} \text { or } \\
c_{1}=c_{1}^{\prime} \text { and } c_{2}<c_{2}^{\prime} \text { or } \\
c_{1}=c_{1}^{\prime} \text { and } c_{2}=c_{2}^{\prime} \ldots c_{n}<c_{n}^{\prime}
\end{array} .\right.
$$

This way we get a total ordering. This approach makes sense when a priority can be placed on the components, but this is of course not the case when dealing with the RGB colour model.

A number of possible orders for colour vectors in the RGB colour model have been proposed in ${ }^{7},{ }^{6},{ }^{5},{ }^{4}$, ${ }^{3}$. In ${ }^{7}$ an $\alpha$-modulus lexicographical order in the RGB colour model is proposed, where the choice of the value for $\alpha$ controls the degree of influence of the first component with regard to the others, and makes the lexicographical order thus more flexible:

$$
c<_{\alpha} c^{\prime} \text { if }\left\{\begin{array}{l}
\left(\frac{c_{1}}{\alpha}\right)<\left(\frac{c_{1}^{\prime}}{\alpha}\right) \text { or } \\
\left(\frac{c_{1}}{\alpha}\right)=\left(\frac{c_{1}^{\prime}}{\alpha}\right) \text { and } c_{2}<c_{2}^{\prime} \text { or } \\
\left(\frac{c_{1}}{\alpha}\right)=\left(\frac{c_{1}^{\prime}}{\alpha}\right) \text { and } c_{2}=c_{2}^{\prime} \ldots c_{n}<c_{n}^{\prime}
\end{array}\right. \text {. }
$$

Because in the RGB colour model no colour R, G or $\mathrm{B}$ plays a dominant role, the maximum and minimum of the three RGB values for every pixel are first calculated. The authors propose an $\alpha$-modulus lexicographical order where the first component is given by, for every colour $c$ in RGB,

$$
\begin{gathered}
I(c)=\beta \cdot\left(0.3 \cdot c_{r}+0.6 \cdot c_{g}+0.1 \cdot c_{b}\right)+ \\
(1-\beta) \cdot\left(\max \left(c_{r}, c_{g}, c_{b}\right)-\min \left(c_{r}, c_{g}, c_{b}\right)\right) .
\end{gathered}
$$


The function $I$ is a combination of the RGB components and the $\max -\min$ of the components, weighted by $\beta$. The other components for ordering are the red, then the green and finally the blue component. After deep test, the authors have found that the value $\beta=0.8$ gives very good visual effects. This order is called $I-R G B_{\alpha}$. In ${ }^{6}$ a reduced ordering in RGB is proposed. The used measurement functions are linear combinations of the tristimulus values, e.g. the luminance image $d(c)=0.299 \cdot c_{r}+0.587 \cdot c_{g}+0.114 \cdot c_{b}$, and the Euclidean norm $d(c)=\sqrt{\left(c_{r}^{2}+c_{g}^{2}+c_{b}^{2}\right)}$. If two different colour values are ordered equally, the output can be chosen based on the position in the structuring element window, but no further analysis or example of this condition is made. New morphological operations are defined. The dilation selects that colour with the largest measure $d_{i}$ and the erosion selects that colour with the smallest $d_{i}$. In ${ }^{5}$ a new approach for the ordering of the RGB model is presented and applied to mathematical morphology, where the adaptation of a linear growing selforganizing network to the three-dimensional colour model allows the definition of an order relationship among colours. In ${ }^{4}$ new colour morphological operators are defined after ordering the RGB colour vectors by using the first principal component analysis. On the basis of this reduced ordering, new infimum and supremum are determined. Using the new infimum and supremum, the fundamental erosion and dilation operators are defined. In $^{3}$ a new set of morphological operators for RGB colour images based on a combination of reduced and conditional ordering is proposed. The RGB colours are transformed into the $C-Y$ colour model, a colour television model. The distance to a reference colour vector, determined by its hue, provides the primary ordering criterion. The defined colour dilation will tend to move towards this reference colour, while the colour erosion will tend to move away from it. But the reference colour must have maximum luminance and maximum saturation to obtain a total ordering of colour vectors, so we can speak of red dilation and blue erosion for example, but white or black cannot be used as reference colour vector. This is of course a disadvantage.

\section{New RGB Colour Ordering}
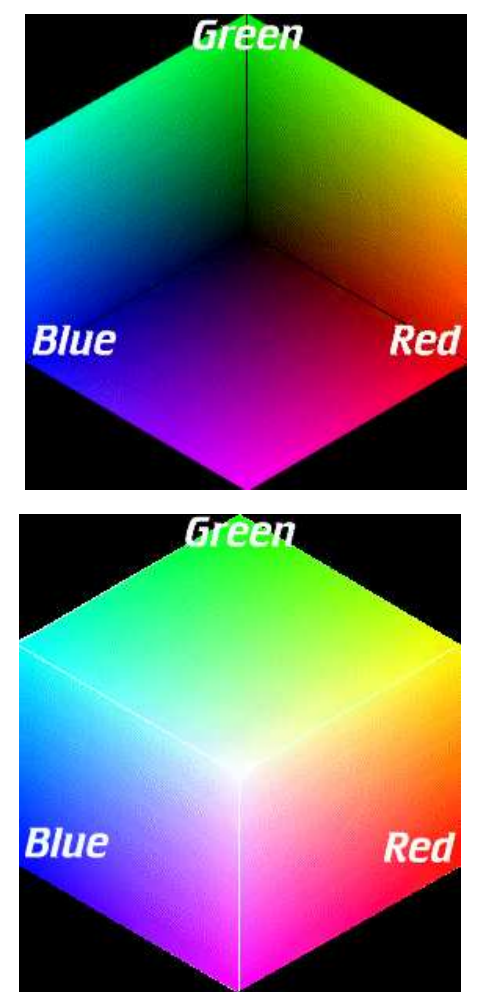

Fig. 2. Representation of the RGB colour model ${ }^{2}$.

On the RGB cube in figure $2^{2}$ we see that colours lying close to black are 'dark' colours while colours lying close to white are 'light' colours. Inspired by this observation we will sort the colours in RGB from 'dark' colours to 'light' colours, with respect to their distance to the centre $m=(1 / 2,1 / 2,1 / 2)$ of the RGB cube (as the middle of the black and white top).

In the RGB cube we consider a plane $V$ through the centre $m=(1 / 2,1 / 2,1 / 2)$ perpendicular to the line $l$ determined by the two points $\operatorname{Bl}(0,0,0)$ and $\mathrm{Wh}(1,1,1)$. The line $l$ is determined as intersection of the two planes $r=g$ and $g=b$. In figure 3 you see the line $l$ and the plane $V$ in the RGB cube. The equation of the plane $V$ with non-zero normal vector $(1,1,1)$ through $(1 / 2,1 / 2,1 / 2)$ is

$$
V: r+g+b-\frac{3}{2}=0 .
$$

For every colour $c=\left(r_{c}, g_{c}, b_{c}\right)$ in RGB we now look if $c$ lies 'below' or 'above' the plane $V$ with respect to its normal vector $(1,1,1)$. So we have to work out the "distance" from $c$ to $V$ as follows

$$
D_{(c, V)}=\frac{\left(r_{c}+g_{c}+b_{c}-3 / 2\right)}{\sqrt{3}} .
$$


If $D_{(c, V)}>0$, then $c$ is on the same side of the plane as the normal vector $(1,1,1)$; if $D_{(c, V)}<0$, then $c$ is on the opposite side; and if $D_{(c, V)}=0$, then $c$ lies in $V$. This way we can distinguish between 'dark' colours lying close to black $\left(D_{(c, V)}<0\right)$ and 'light' colours lying close to white $\left(D_{(c, V)}>0\right)$, where we will rank 'dark' colours lower than 'light' colours. The plane $V$ 'divides' the RGB cube into two similar parts.

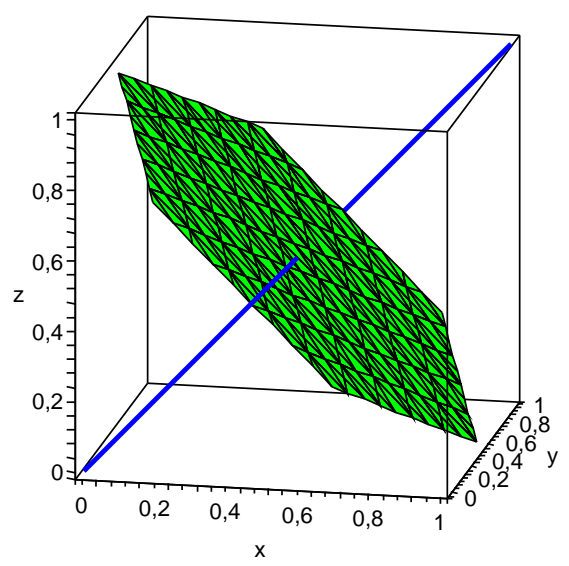

Fig. 3. The RGB colour cube with the plane $V$ (in green) and the line $l$ (in blue).

Let $c=\left(r_{c}, g_{c}, b_{c}\right)$ and $c^{\prime}=\left(r_{c^{\prime}}, g_{c^{\prime}}, b_{c^{\prime}}\right)$ be two colours in RGB. We will consider the following cases for the ranking of $c$ and $c^{\prime}$ :

$\mathbf{1}^{\text {st }}$ case: The two colours $c$ and $c^{\prime}$ are not lying on the same side w.r.t. $V$

1.1. $c$ lies under $V$ and $c^{\prime}$ lies above $V$, i.e., $D_{(c, V)}<0$ and $D_{\left(c^{\prime}, V\right)}>0$

1.2. $c$ lies in $V$ and $c^{\prime}$ lies above $V$, i.e., $D_{(c, V)}=0$ and $D_{\left(c^{\prime}, V\right)}>0$

1.3. $c$ lies under $V$ and $c^{\prime}$ lies in $V$, i.e., $D_{(c, V)}<0$ and $D_{\left(c^{\prime}, V\right)}=0$.

In these three subcases $c$ is ranked lower than $c^{\prime}$, i.e., $c<_{R G B} c^{\prime}$.

$\mathbf{2}^{\text {nd }}$ case: The two colours $c$ and $c^{\prime}$ are lying on the same side w.r.t. $V$

2.1. $c$ and $c^{\prime}$ are lying above $V$, i.e., $D_{(c, V)}>0$ and $D_{\left(c^{\prime}, V\right)}>0 ; c$ and $c^{\prime}$ lie both close to white
All colours above $V$ are considered to be 'light' colours lying close to white. Because we want to determine which of the two colours $c$ and $c^{\prime}$ is the 'lightest' colour, we slice the cube by looking at the distance to $V$ :

$$
\begin{gathered}
D(c, V)=\left|r_{c}+g_{c}+b_{c}-3 / 2\right| / \sqrt{3}=\left|D_{(c, V)}\right| \\
D\left(c^{\prime}, V\right)=\left|r_{c^{\prime}}+g_{c^{\prime}}+b_{c^{\prime}}-3 / 2\right| / \sqrt{3}=\left|D_{\left(c^{\prime}, V\right)}\right|
\end{gathered}
$$

where the colours $c$ and $c^{\prime}$ are then ordered accordingly to their distance with respect to $V$.

2.1.1. $D(c, V)<D\left(c^{\prime}, V\right): c$ is a 'darker' colour than $c^{\prime}$ so that we rank $c$ lower than $c^{\prime}$, i.e., $c<_{R G B} c^{\prime}$.

2.1.2. $D(c, V)>D\left(c^{\prime}, V\right): c$ is a 'lighter' colour than $c^{\prime}$ so that we rank $c$ higher than $c^{\prime}$, i.e., $c>_{R G B} c^{\prime}$.

2.1.3. $D(c, V)=D\left(c^{\prime}, V\right) \rightarrow$ the distance to $m$ is taken into account:

If $\left(D_{(c, V)}>0\right.$ and $\left.D_{\left(c^{\prime}, V\right)}>0\right)$ and $(D(c, V)=$ $\left.D\left(c^{\prime}, V\right)\right)$, thus $D_{(c, V)}=D_{\left(c^{\prime}, V\right)}>0$, that is, if the two colours $c$ and $c^{\prime}$ lie on the same plane $W_{c, c^{\prime}}$ parallel to $V$ at distance $D(c, V)=D\left(c^{\prime}, V\right)$, then we determine the distance from $c$ and $c^{\prime}$ to the centre $m$ of the cube as

$$
\begin{gathered}
D(c, m)=\sqrt{\left(r_{c}-\frac{1}{2}\right)^{2}+\left(g_{c}-\frac{1}{2}\right)^{2}+\left(b_{c}-\frac{1}{2}\right)^{2}} \\
D\left(c^{\prime}, m\right)=\sqrt{\left(r_{c^{\prime}}-\frac{1}{2}\right)^{2}+\left(g_{c^{\prime}}-\frac{1}{2}\right)^{2}+\left(b_{c^{\prime}}-\frac{1}{2}\right)^{2}}
\end{gathered}
$$

We sort the colours w.r.t. their distance to $m$, that is,

2.1.3.1. $D(c, m)<D\left(c^{\prime}, m\right): c$ is ranked lower than $c^{\prime}$, i.e., $c<_{R G B} c^{\prime}$.

2.1.3.2. $D(c, m)>D\left(c^{\prime}, m\right): c$ is ranked higher than $c^{\prime}$, i.e., $c>_{R G B} c^{\prime}$.

2.1.3.3. $D(c, m)=D\left(c^{\prime}, m\right)$, see subcase 2.3 .

2.2. $c$ and $c^{\prime}$ are lying below $V$, i.e., $D_{(c, V)}<0$ and $D_{\left(c^{\prime}, V\right)}<0 ; c$ and $c^{\prime}$ lie both close to black

Here we can make an analogous reasoning as in case 2.1. Again we first take the distance to $V$ into account:

2.2.1. $D(c, V)>D\left(c^{\prime}, V\right)$ : we rank $c$ lower than $c^{\prime}$, i.e., $c<_{R G B} c^{\prime}$. 
2.2.2. $D(c, V)<D\left(c^{\prime}, V\right)$ : we rank $c$ higher than $c^{\prime}$, i.e., $c>_{R G B} c^{\prime}$.

2.2.3. $D(c, V)=D\left(c^{\prime}, V\right) \rightarrow$ the distance to $m$ is taken into account:

2.2.3.1. $D(c, m)>D\left(c^{\prime}, m\right): c$ is ranked lower than $c^{\prime}$, i.e., $c<_{R G B} c^{\prime}$.

2.2.3.2. $D(c, m)<D\left(c^{\prime}, m\right): c$ is ranked higher than $c^{\prime}$, i.e., $c>_{R G B} c^{\prime}$.

2.2.3.3. $D(c, m)=D\left(c^{\prime}, m\right)$, see subcase 2.3 .

2.3. In this part we can take the two subcases 2.1.3.3. and 2.2.3.3. together.

If for $c$ and $c^{\prime}$ hold that $D_{(c, V)}<0$ and $D_{\left(c^{\prime}, V\right)}<0$ and $D(c, V)=D\left(c^{\prime}, V\right)$ and $D(c, m)=D\left(c^{\prime}, m\right)$ or $D_{(c, V)}>0$ and $D_{\left(c^{\prime}, V\right)}>0$ and $D(c, V)=$ $D\left(c^{\prime}, V\right)$ and $D(c, m)=D\left(c^{\prime}, m\right)$, then both colours $c$ and $c^{\prime}$ lie not only on the same plane $W_{c, c^{\prime}}$ parallel to $V$, but also on the same sphere $S$ at distance $D(c, m)=D\left(c^{\prime}, m\right)$ from the centre $m$. If $D_{(c, V)}=D_{\left(c^{\prime}, V\right)}<0$, the sphere $S$ lies below the plane $V$; if $D_{(c, V)}=D_{\left(c^{\prime}, V\right)}>0$, the sphere $S$ lies above the plane $V$. Consequently, $c$ and $c^{\prime}$ lie on a circle $C_{c, c^{\prime}}$ in $W_{c, c^{\prime}}$ parallel to $V$ with centre on the line $l$. All these colour hues are considered to be equally important, so that we really have to choose one out of these two colours to be the smallest (or largest) colour. And therefore we will order $c$ and $c^{\prime}$ by defining an angle $\theta^{\prime}$ in $C_{c, c^{\prime}}$.

If we cut the plane $W_{c, c^{\prime}}$ with the line $l$, we get the centre of our circle $C_{c, c^{\prime}}$. The plane $W_{c, c^{\prime}}$ is parallel to $V$, and thus has the same normal vector as $V$, so we get

$$
W_{c, c^{\prime}}: r+g+b-d_{W}=0
$$

with $d_{W}=D_{W} \cdot \sqrt{3}$, where $D_{W}$ is the distance of $W$ from the origin $(0,0,0)$.

$$
D_{W}=D_{V}+D_{\left(W_{c, c^{\prime}}, V\right)}
$$

where $D_{V}$ is the distance of $(0,0,0)$ to the plane $V$, $D_{V}=\sqrt{\frac{3}{4}}=\frac{\sqrt{3}}{2}$; and $D_{\left(W_{c, c^{\prime}}, V\right)}$ is the "distance" between the two planes $V$ and $W_{c, c^{\prime}}, D_{\left(W_{c, c^{\prime}}, V\right)}=$ $D_{(c, V)}=D_{\left(c^{\prime}, V\right)}$.

The centre $a=\left(r_{a}, g_{a}, b_{a}\right)$, where $r_{a}=g_{a}=b_{a}$, of the circle $C_{c, c^{\prime}}$ has to satisfy

$$
\left\{\begin{array}{ccc}
r+g+b-d_{W} & = & 0 \\
r & = & g \\
g & =
\end{array},\right.
$$

or thus $a=\left(d_{W} / 3, d_{W} / 3, d_{W} / 3\right)$. For the radius $r$ of $C_{c, c^{\prime}}$ we get

$$
\begin{gathered}
r=d(c, a)=d\left(c^{\prime}, a\right)= \\
\sqrt{\left(r_{c}-d_{W} / 3\right)^{2}+\left(g_{c}-d_{W} / 3\right)^{2}+\left(b_{c}-d_{W} / 3\right)^{2}} .
\end{gathered}
$$

Now we will define an angle $\theta_{c}^{\prime}$ from the centre $a$ of $C_{c, c^{\prime}}$ for every colour $c$ lying on $C_{c, c^{\prime}}$ :

All lines through $a$ in the plane $W_{c, c^{\prime}}$ are perpendicular to $l$. We want to choose a fixed 'direction' that is the same for every plane $W_{c, c^{\prime}}$, and thus independent of $W_{c, c^{\prime}}$, through which we define the angle $\theta_{c}^{\prime}$. And therefore we cut the plane $W_{c, c^{\prime}}$ with the upper plane of the cube $b=1$ so that we get a line $W W_{c, c^{\prime}}$ :

$$
\left\{\begin{array}{ccc}
r+g+b-d_{W} & = & 0 \\
b & = & 1
\end{array}\right.
$$

or

$$
W W_{c, c^{\prime}}: r+g+\left(1-d_{W}\right)=0 .
$$

Next, we consider the line $X_{c, c^{\prime}}$ through the centre $a$ perpendicular to the line $W W_{c, c^{\prime}}$. We determine the equation of $X_{c, c^{\prime}}$ as the intersection of two planes, namely the plane $W_{c, c^{\prime}}$ and the plane through the line $l$ perpendicular to the line $W W_{c, c^{\prime}}$, which has equation $r=g$. So that we obtain for $X_{c, c^{\prime}}$

$$
\left\{\begin{array}{cc}
r & =g \\
r+g+b-d_{W} & =0
\end{array} .\right.
$$

We know that $X_{c, c^{\prime}}$ goes through the point $a$ and when we cut $X_{c, c^{\prime}}$ with the line $W W_{c, c^{\prime}}$, we get another point $\gamma$ of $X_{c, c^{\prime}}$,

$$
\left\{\begin{array}{ccc}
r & = & g \\
r+g+b-d_{W} & = & 0 \\
r+g+\left(1-d_{W}\right) & = & 0
\end{array},\right.
$$

or thus $\gamma$ has coordinates $\left(\frac{d_{W}-1}{2}, \frac{d_{W}-1}{2}, 1\right)$. The line $X_{c, c^{\prime}}$ gives us the fixed chosen direction, which can be determined for every plane $W_{c, c^{\prime}}$ in the same way, to define $\theta_{c}^{\prime}$.

Accordingly, we first define for a colour $c$ lying on the circle $C_{c, c^{\prime}}$ an angle $\theta_{c}$ determined by

$$
\theta_{c}=\arcsin \frac{d_{c}}{d(a, c)}(\in[0, \pi / 2])
$$

$d(a, c)=\sqrt{\left(r_{c}-\frac{d_{W}}{3}\right)^{2}+\left(g_{c}-\frac{d_{W}}{3}\right)^{2}+\left(b_{c}-\frac{d_{W}}{3}\right)^{2}}$, the distance between $a$ and $c$, and $d_{c}$ the perpendicular distance between $c$ and the line $X_{c, c^{\prime}}$ through 
the two points $a$ and $\gamma$. Let $a c\left(r_{a c}, g_{a c}, b_{a c}\right)=$ $a\left(r_{a}, g_{a}, b_{a}\right)-c\left(r_{c}, g_{c}, b_{c}\right)$ and $\gamma a\left(r_{\gamma a}, g_{\gamma a}, b_{\gamma a}\right)=$ $\gamma\left(r_{\gamma}, g_{\gamma}, b_{\gamma}\right)-a\left(r_{a}, g_{a}, b_{a}\right)$. The distance $d_{c}$ is then given by

$$
d_{c}^{2}=\frac{|a c|^{2}|\gamma a|^{2}-((a c) \cdot(\gamma a))^{2}}{|\gamma a|^{2}}
$$

or

$$
\begin{aligned}
& d_{c}^{2}=\left(\left(r_{a c}^{2}+g_{a c}^{2}+b_{a c}^{2}\right) \cdot\left(r_{\gamma a}^{2}+g_{\gamma a}^{2}+b_{\gamma a}^{2}\right)-\right. \\
& \left.\left(r_{a c} \cdot r_{\gamma a}+g_{a c} \cdot g_{\gamma a}+b_{a c} \cdot b_{\gamma a}\right)^{2}\right) /\left(r_{\gamma a}^{2}+g_{\gamma a}^{2}+b_{\gamma a}^{2}\right) .
\end{aligned}
$$

Now we choose a 'direction' for $\theta_{c}$ and so define the angle $\theta_{c}^{\prime}$ as

if $b_{c}>b_{a}(c$ lies above $a)$

if $r_{c}>g_{c}$

$$
\theta_{c}^{\prime}=\theta_{c}
$$

else if $g_{c}>r_{c}$

$$
\theta_{c}^{\prime}=2 \pi-\theta_{c}
$$

else if $r_{c}=g_{c}$

$$
\theta_{c}^{\prime}=\theta_{c}=0
$$

else if $b_{c}<b_{a}(c$ lies below $a)$

if $r_{c}>g_{c}$

$$
\theta_{c}^{\prime}=\pi-\theta_{c}
$$

else if $g_{c}>r_{c}$

$$
\theta_{c}^{\prime}=\pi+\theta_{c}
$$

else if $r_{c}=g_{c}$

$$
\theta_{c}^{\prime}=\pi
$$

else if $b_{c}=b_{a}$

$$
\begin{aligned}
& \text { if } r_{c}>g_{c} \\
& \quad \theta_{c}^{\prime}=\theta_{c}=\pi / 2
\end{aligned}
$$

else if $g_{c}>r_{c}$

$$
\theta_{c}^{\prime}=3 \pi / 2
$$

else if $r_{c}=g_{c}$

$$
\text { impossible. }
$$

In this last step, where for the two colours $c$ and $c^{\prime}$ hold that $D_{(c, V)}=D_{\left(c^{\prime}, V\right)} \neq 0$ and $D(c, m)=$ $D\left(c^{\prime}, m\right)$, we order their corresponding angles $\theta_{c}^{\prime}$ and $\theta_{c^{\prime}}^{\prime}$ as follows: if: $\left(\left(\theta_{c}^{\prime} \in\left[0, \pi\left[\right.\right.\right.\right.$ and $\theta_{c^{\prime}}^{\prime} \in\left[0, \pi[)\right.$ or $\left(\theta_{c}^{\prime} \in[0, \pi[\right.$ and $\theta_{c^{\prime}}^{\prime} \in[\pi, 2 \pi[))$ and $\theta_{c}^{\prime}<\theta_{c^{\prime}}^{\prime}$

then: $\theta_{c}^{\prime}<_{\theta} \theta_{c^{\prime}}^{\prime}$

if: $\theta_{c}^{\prime} \in\left[\pi, 2 \pi\left[\right.\right.$ and $\theta_{c^{\prime}}^{\prime} \in\left[\pi, 2 \pi\left[\right.\right.$ and $2 \pi-\theta_{c}^{\prime}<$ $2 \pi-\theta_{c^{\prime}}^{\prime}\left(\right.$ or thus $\left.\theta_{c}^{\prime}>\theta_{c^{\prime}}^{\prime}\right)$

then: $\theta_{c}^{\prime}<_{\theta} \theta_{c^{\prime}}^{\prime}$.

2.4. $c$ and $c^{\prime}$ are lying in $V$, i.e., $D_{(c, V)}=D_{\left(c^{\prime}, V\right)}=$ 0

Because we want our ordering to be compatible with the complement $c o$, we order the colours $c$ and $c^{\prime}$ here as follows:

2.4.1. $D(c, m) \cos \left(\theta_{c}^{\prime}\right)<D\left(c^{\prime}, m\right) \cos \left(\theta_{c^{\prime}}^{\prime}\right)$ : we rank $c$ lower than $c^{\prime}$, i.e., $c<_{R G B} c^{\prime}$.

2.4.2. $D(c, m) \cos \left(\theta_{c}^{\prime}\right)>D\left(c^{\prime}, m\right) \cos \left(\theta_{c^{\prime}}^{\prime}\right)$ : we rank $c$ higher than $c^{\prime}$, i.e., $c>_{R G B} c^{\prime}$.

2.4.3. $D(c, m) \cos \left(\theta_{c}^{\prime}\right)=D\left(c^{\prime}, m\right) \cos \left(\theta_{c^{\prime}}^{\prime}\right)$ and $D(c, m) \sin \left(\theta_{c}^{\prime}\right)<D\left(c^{\prime}, m\right) \sin \left(\theta_{c^{\prime}}^{\prime}\right)$ : we rank $c$ lower than $c^{\prime}$, i.e., $c<_{R G B} c^{\prime}$.

2.4.4. $D(c, m) \cos \left(\theta_{c}^{\prime}\right)=D\left(c^{\prime}, m\right) \cos \left(\theta_{c^{\prime}}^{\prime}\right)$ and $D(c, m) \sin \left(\theta_{c}^{\prime}\right)>D\left(c^{\prime}, m\right) \sin \left(\theta_{c^{\prime}}^{\prime}\right)$ : we rank $c$ higher than $c^{\prime}$, i.e., $c>_{R G B} c^{\prime}$.

\section{Definition of $\leq_{R G B}$}

Consider two colours $c(r, g, b)$ and $c^{\prime}\left(r^{\prime}, g^{\prime}, b^{\prime}\right)$ in RGB, then it holds

$$
\begin{aligned}
& c<_{R G B} c^{\prime} \Leftrightarrow\left(D_{(c, V)}<0 \text { and } D_{\left(c^{\prime}, V\right)}>0\right) \\
& \text { or }\left(D_{(c, V)}=0 \text { and } D_{\left(c^{\prime}, V\right)}>0\right) \\
& \text { or }\left(D_{(c, V)}<0 \text { and } D_{\left(c^{\prime}, V\right)}=0\right) \\
& \text { or }\left(D_{(c, V)}<0 \text { and } D_{\left(c^{\prime}, V\right)}<0\right. \text { and } \\
& \left.\quad D(c, m)>D\left(c^{\prime}, m\right)\right) \\
& \quad \text { or }\left(D_{(c, V)}>0 \text { and } D_{\left(c^{\prime}, V\right)}>0\right. \text { and } \\
& \left.\quad D(c, m)<D\left(c^{\prime}, m\right)\right) \\
& \text { or }\left(D_{(c, V)}<0 \text { and } D_{\left(c^{\prime}, V\right)}<0\right. \text { and } \\
& D(c, m)=D\left(c^{\prime}, m\right) \text { and } \\
& \left.D(c, V)>D\left(c^{\prime}, V\right)\right) \\
& \text { or }\left(D_{(c, V)}>0 \text { and } D_{\left(c^{\prime}, V\right)}>0\right. \text { and } \\
& D(c, m)=D\left(c^{\prime}, m\right) \text { and } \\
& \left.D(c, V)<D\left(c^{\prime}, V\right)\right) \\
& \text { or }\left(D_{(c, V)}=D_{\left(c^{\prime}, V\right)} \neq 0\right. \text { and } \\
& D(c, m)=D\left(c^{\prime}, m\right) \text { and } \\
& \left.\theta_{c}^{\prime}<{ }_{\theta} \theta_{c^{\prime}}^{\prime}\right)
\end{aligned}
$$




$$
\begin{gathered}
\text { or }\left(D_{(c, V)}=D_{\left(c^{\prime}, V\right)}=0\right. \text { and } \\
\left.D(c, m) \cos \left(\theta_{c}^{\prime}\right)<D\left(c^{\prime}, m\right) \cos \left(\theta_{c^{\prime}}^{\prime}\right)\right) \\
\text { or }\left(D_{(c, V)}=D_{\left(c^{\prime}, V\right)}=0\right. \text { and } \\
D(c, m) \cos \left(\theta_{c}^{\prime}\right)=D\left(c^{\prime}, m\right) \cos \left(\theta_{c^{\prime}}^{\prime}\right) \\
\text { and } \\
\left.D(c, m) \sin \left(\theta_{c}^{\prime}\right)<D\left(c^{\prime}, m\right) \sin \left(\theta_{c^{\prime}}^{\prime}\right)\right) \\
c>_{R G B} c^{\prime} \Leftrightarrow c^{\prime}<_{R G B} c \\
c=R G B c^{\prime} \Leftrightarrow D_{(c, V)}=D_{\left(c^{\prime}, V\right)} \text { and } \\
D(c, m)=D\left(c^{\prime}, m\right) \text { and } \theta_{c}^{\prime}=\theta_{c^{\prime}}^{\prime} \\
c \leq R G B c^{\prime} \Leftrightarrow c<_{R G B} c^{\prime} \text { or } c^{\prime}=R G B
\end{gathered}
$$

\section{Definition of the Complement co}

In the RGB colour model we want the definition of the complement co of a colour to fulfill the property that the RGB and CMY colours are complementary colours, $c o($ red $)=$ cyan, $c o($ green $)=$ magenta, $c o$ (blue) $=$ yellow and vice versa, so if $c\left(r_{c}, g_{c}, b_{c}\right)$ is a colour in RGB, we define the complement $c o(c)$ of $c$ as $(c o(c))(r, g, b) \stackrel{\text { def }}{=} \mathbf{1}_{R G B}-c$ with $r \stackrel{\text { def }}{=} 1-r_{c}, g \stackrel{\text { def }}{=}$ $1-g_{c}, b \stackrel{\text { def }}{=} 1-b_{c}$.

\section{Properties of $\leq_{R G B}$}

We can show (see Appendix) that our new ordering $\leq_{R G B}$ is reflexive, antisymmetric and transitive, and that our new ordering $\leq_{R G B}$ in RGB is compatible with the complement $c o$, i.e., for all colours $c, c^{\prime}$ in $R G B$ :

$$
c \leq_{R G B} c^{\prime} \Leftrightarrow c o(c) \geq_{R G B} c o\left(c^{\prime}\right) .
$$

\section{Associated Minimum and Maximum Opera- tors}

The minimum (maximum) of a set $S$ of $n$ colours $c_{1}\left(r_{1}, g_{1}, b_{1}\right), \ldots, c_{n}\left(r_{n}, g_{n}, b_{n}\right)$ in $R G B$ is the colour $c_{\alpha} \in S$ wherefore $c_{\alpha} \leq_{R G B} \quad c_{i}\left(\begin{array}{lll}c_{\alpha} & \geq_{R G B} & c_{i}\end{array}\right)$, $\forall i=1 \ldots n$.

$\left(R G B, \leq_{R G B}\right)$ is a poset, and what's more, by definition of the order relation $\leq_{R G B}$, it holds that

$$
\left(\forall c, c^{\prime} \in R G B\right)\left(c \leq_{R G B} c^{\prime} \text { or } c^{\prime} \leq_{R G B} c\right),
$$

so that $\left(R G B, \leq_{R G B}\right)$ is a totally ordered set $\left(\max _{R G B}\left(c, c^{\prime}\right)\right.$ and $\min _{R G B}\left(c, c^{\prime}\right)$ exist for all $c, c^{\prime} \in$
$R G B)$, and thus a lattice. The greatest element in $\left(R G B, \leq_{R G B}\right)$ is $\mathbf{1}=(1,1,1)$ and the smallest element is $\mathbf{0}=(0,0,0)$, so we get a bounded complete lattice.

\section{Morphological Image Interpolation to Magnify Colour Images with Sharp Edges}

In ${ }^{8}$ and ${ }^{1}$ we have presented a new image interpolation method, based on mathematical morphology, to magnify images with sharp edges. Our method was developed for binary images and colour images with a restricted number of colours. Now we have extended our magnification method towards all colour images with sharp edges, where we have used the new colour vector ordering $\leq_{R G B}$. First we use the pixel replicated or nearest neighbour interpolated image $O$ of an original image $X$ as a first 'trivial' interpolation step. To detect the unwanted jaggies in the blown up image $O$, we determine the inner edge-image (intern morphological gradient) of $O$ and of the complementary image $c o(O)$ of $O$ by a full 3 by 3 structuring element and then apply the hit-or-miss operator to obtain the positions of the corner edge pixels. We have used different kinds of structuring elements in the hit-or-miss transformation to detect all corners and depending on the kind of corner we detect, we will smooth the corners. Our proposed corner transformation method is a trade-off between blur and jaggies.

Now, we need the proposed ordering $\leq_{R G B}$, in particular $\min _{R G B}$ and $\max _{R G B}$, to determine the intern morphological gradients of $O$ and $c o(O)$ by a structuring element, where it is important and even necessary that the used colour vector ordering $\leq_{R G B}$ is compatible with the complement co to get 'supplementary' inner edge-images for $O$ and $c o(O)$.

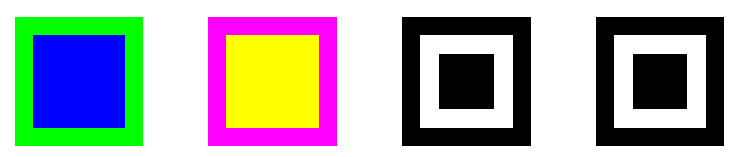



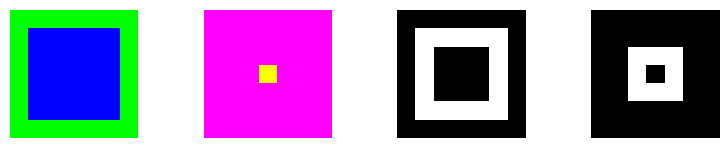

Fig. 4. From left to right: above: possible t-erosions of $O$ and $c o(O)$, and the corresponding inner edge images, when using an ordering that is not compatible with complement co, below: the t-'colour' erosions of $O$ and $c o(O)$, and their corresponding intern morphological gradients, using $\leq_{R G B}$.

In figure 4 you see an original image $O$ and its complement $c o(O)$. If we use an ordering that is not compatible with the complement $c o$, we can get results as in figure 4 (upper line) for the inner edge-image of $O$ and $c o(O)$, where it is impossible to detect corners using the hit-or-miss transform. Figure 4 (lower line) shows the t-'colour' erosions ${ }^{8}$ and ${ }^{1}$ of $O$ and $c o(O)$ and their corresponding induced intern morphological gradients by a full 3 by 3 structuring element. Figure 5 and 6 illustrate some results of our interpolation method. Our method provides very good results. Improvements in visual quality can be noticed: unwanted jaggies have been removed so that edges have become smoother and the edges are very sharp and clear.

\section{Conclusions}

This paper presents a new ordering $\leq_{R G B}$ for colours in the RGB colour model so that we obtain a complete lattice $\left(R G B, \leq_{R G B}\right)$. We demonstrated as application the magnification of colour images in RGB with sharp edges, making use of mathematical morphology and the proposed ordering $\leq_{R G B}$. Our method gives very good results and improves the visual quality of magnified colour images with 'vague' edges. The problem of interpolating an image is the introduction of unwanted jagged edges in the blown up image. We have developed a new approach to avoid these jaggies, which is a trade-off between blur and jaggies. Our method removes unwanted jaggies so that edges become smooth and very sharp and clear.
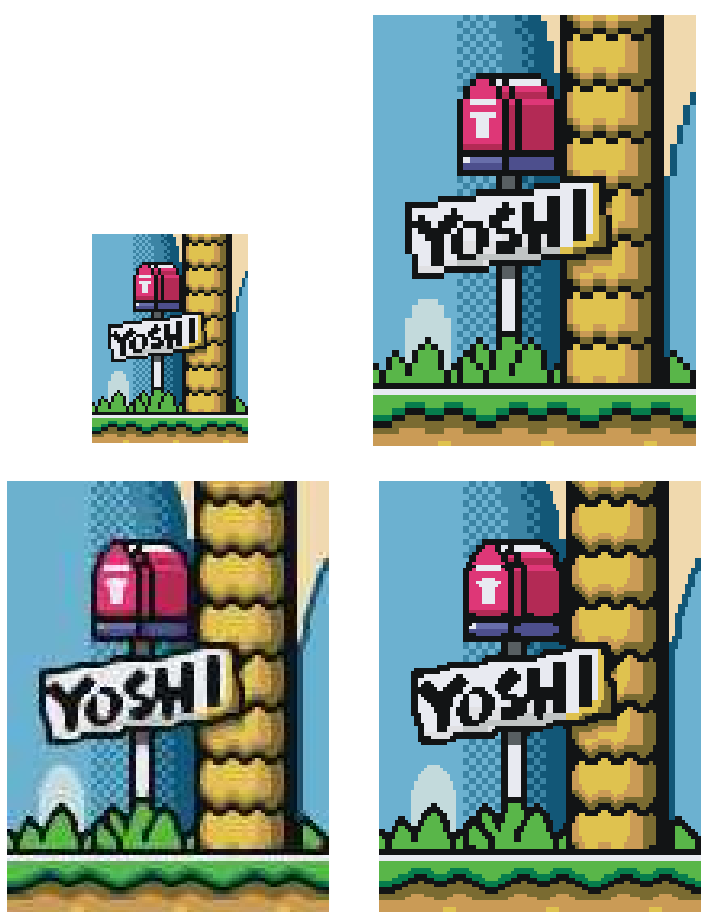

Fig. 5. At the top: the original 'mailbox' image (left) and the nearest neighbour interpolated image for magnification by a factor 2 (right), at the bottom: the result of our new morphological interpolation method (left) and our method, giving as result a colour image with no new colours (right).
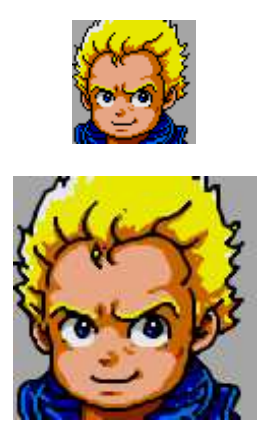

Fig. 6. At the top: the original 'man' image (left) and the pixel replicated image for magnification by a factor 2

(right), at the bottom: our new morphological interpolation method (left) and the result of our method with no new colours (right).

\section{Appendix A}

We examine some properties of our new ordering $\leq_{R G B}$.

1. Reflexive: $(\forall a \in R G B)\left(a \leq_{R G B} a\right)$. OK.

2. Antisymmetric: $(\forall a, b \in R G B)\left(a \leq_{R G B} b\right.$ and 
$\left.b \leq_{R G B} a \stackrel{?}{\Rightarrow} a={ }_{R G B} b\right)$

\section{Proof}

Suppose that $a \neq_{R G B} b$.

$1.1\left(D_{(a, V)}<0\right.$ and $\left.D_{(b, V)}>0\right)$ and $b<_{R G B} a$

From the definition of $<_{R G B}$ would follow: $D_{(a, V)}>0$, a contradiction.

$1.2\left(D_{(a, V)}=0\right.$ and $\left.D_{(b, V)}>0\right)$ and $b<_{R G B} a$

From the definition of $<_{R G B}$ would follow: $D_{(a, V)}>0$, a contradiction.

$1.3\left(D_{(a, V)}<0\right.$ and $\left.D_{(b, V)}=0\right)$ and $b<_{R G B} a$

From the definition of $<_{R G B}$ would follow: $D_{(a, V)} \geq 0$, a contradiction.

2.1.1 $\left(D_{(a, V)}>0\right.$ and $D_{(b, V)}>0$ and $D(a, V)<$ $D(b, V))$ and $b<_{R G B} a$

From the definition of $<_{R G B}$ would follow: $D_{(a, V)}>0$ and $D(b, V) \leq D(a, V)$, a contradiction.

2.2.1 $\left(D_{(a, V)}<0\right.$ and $D_{(b, V)}<0$ and $D(a, V)>$ $D(b, V))$ and $b<_{R G B} a$

From the definition of $<_{R G B}$ would follow:

(a) $D_{(b, V)}<0$ and $D_{(a, V)} \geq 0$, a contradiction.

(b) $D_{(b, V)}<0$ and $D_{(a, V)}<0$ and $D(b, V) \geq$ $D(a, V)$, a contradiction.

2.1.3 $\left(D_{(a, V)}>0\right.$ and $D_{(b, V)}>0$ and $D(a, V)=$ $D(b, V)$ and $D(a, m)<D(b, m))$ and $b<_{R G B}$ a

From the definition of $<_{R G B}$ would follow: $D(b, m) \leq D(a, m)$, a contradiction.

2.2.3 $\left(D_{(a, V)}<0\right.$ and $D_{(b, V)}<0$ and $D(a, V)=$ $D(b, V)$ and $D(a, m)>D(b, m))$ and $b<_{R G B}$ a

From the definition of $<_{R G B}$ would follow:

(a) $D_{(b, V)}<0$ and $D_{(a, V)} \geq 0$, a contradiction. (b) $D_{(b, V)}<0$ and $D_{(a, V)}<0$ and $D(a, V)=$ $D(b, V)$ and $D(b, m) \geq D(a, m)$, a contradiction.

$2.3\left(D_{(a, V)}=D_{(b, V)} \neq 0\right.$ and $D(a, m)=D(b, m)$ and $\left.\theta_{a}^{\prime}<_{\theta} \theta_{b}^{\prime}\right)$ and $b<_{R G B} a$

From the definition of $<_{R G B}$ would follow: $\theta_{b}^{\prime}<{ }_{\theta} \theta_{a}^{\prime}$, a contradiction.

2.4.1 $\left(D_{(a, V)}=D_{(b, V)}=0\right.$ and $D(a, m) \cos \left(\theta_{a}^{\prime}\right)<$ $\left.D(b, m) \cos \left(\theta_{b}^{\prime}\right)\right)$ and $b<_{R G B} a$

From the definition of $<_{R G B}$ would follow: $D(b, m) \cos \left(\theta_{b}^{\prime}\right) \leq D(a, m) \cos \left(\theta_{a}^{\prime}\right)$, a contradiction.

2.4.3 $\left(D_{(a, V)}=D_{(b, V)}=0\right.$ and $D(a, m) \cos \left(\theta_{a}^{\prime}\right)=$ $D(b, m) \cos \left(\theta_{b}^{\prime}\right)$ and $D(a, m) \sin \left(\theta_{a}^{\prime}\right)<D(b, m)$ $\left.\sin \left(\theta_{b}^{\prime}\right)\right)$ and $b<_{R G B} a$

From the definition of $<_{R G B}$ would follow: $D(b, m) \sin \left(\theta_{b}^{\prime}\right)<D(a, m) \sin \left(\theta_{a}^{\prime}\right)$, a contradiction.

$$
\begin{aligned}
& \Rightarrow \quad D_{(a, V)}=D_{(b, V)} \text { and } D(a, m)=D(b, m) \text { and } \\
& \quad \theta_{a}^{\prime}=\theta_{b}^{\prime} \\
& \Rightarrow \quad a=_{R G B} b .
\end{aligned}
$$

3. Transitive: $(\forall a, b, c \in R G B)\left(a \leq_{R G B} b\right.$ and $\left.b \leq_{R G B} c \stackrel{?}{\Rightarrow} a \leq_{R G B} c\right)$

\section{Proof}

Let $a={ }_{R G B} \quad b$ and $b \leq_{R G B} \quad c$, then it holds that $a \leq_{R G B} c$. Let $a \leq_{R G B} b$ and $b={ }_{R G B} c$, then it holds that $a \leq_{R G B} c$. So suppose that $a \neq_{R G B} b$ and $b \neq_{R G B} c$, thus $a<_{R G B} b$ and $b<_{R G B} c$.

$1.1\left(D_{(a, V)}<0\right.$ and $\left.D_{(b, V)}>0\right)$ and $b<_{R G B} c$.

From the definition of $<_{R G B}$ would follow:

$$
\begin{aligned}
& D_{(b, V)}>0 \text { and } D_{(c, V)}>0 \\
& \Rightarrow D_{(a, V)}<0 \text { and } D_{(c, V)}>0
\end{aligned}
$$

$\Rightarrow a<_{R G B} c$.

$1.2\left(D_{(a, V)}=0\right.$ and $\left.D_{(b, V)}>0\right)$ and $b<_{R G B} c$.

From the definition of $<_{R G B}$ would follow:

$$
\begin{aligned}
& D_{(b, V)}>0 \text { and } D_{(c, V)}>0 \\
& \Rightarrow D_{(a, V)}=0 \text { and } D_{(c, V)}>0
\end{aligned}
$$


$\Rightarrow a<_{R G B} c$

$1.3\left(D_{(a, V)}<0\right.$ and $\left.D_{(b, V)}=0\right)$ and $b<_{R G B} c$.

From the definition of $<_{R G B}$ would follow:

$$
\begin{aligned}
& D_{(b, V)}=0 \text { and } D_{(c, V)} \geq 0 \\
& \Rightarrow D_{(a, V)}<0 \text { and } D_{(c, V)} \geq 0
\end{aligned}
$$

$\Rightarrow a<_{R G B} c$

2.1.1 $\left(D_{(a, V)}>0\right.$ and $D_{(b, V)}>0$ and $D(a, V)<$ $D(b, V))$ and $b<_{R G B} c$.

From the definition of $<_{R G B}$ would follow:

$D_{(b, V)}>0$ and $D_{(c, V)}>0$ and $D(b, V) \leq$ $D(c, V)$

$\Rightarrow D_{(a, V)}>0$ and $D_{(c, V)}>0$ and $D(a, V)<D(c, V)$

$\Rightarrow a<_{R G B} c$.

2.2.1 $\left(D_{(a, V)}<0\right.$ and $D_{(b, V)}<0$ and $D(a, V)>$ $D(b, V))$ and $b<_{R G B} c$.

From the definition of $<_{R G B}$ would follow:

(a) $D_{(b, V)}<0$ and $D_{(c, V)} \geq 0$

$\Rightarrow D_{(a, V)}<0$ and $D_{(c, V)} \geq 0$

(b) $D_{(b, V)}<0$ and $D_{(c, V)}<0$ and $D(b, V) \geq$ $D(c, V)$

$\Rightarrow D_{(a, V)}<0$ and $D_{(c, V)}<0$ and $D(a, V)>D(c, V)$

$\Rightarrow a<_{R G B} c$.

2.1.3 $\left(D_{(a, V)}>0\right.$ and $D_{(b, V)}>0$ and $D(a, V)=$ $D(b, V)$ and $D(a, m)<D(b, m))$ and $b<_{R G B}$ c.

From the definition of $<_{R G B}$ would follow that:

(a) $D_{(b, V)}>0$ and $D_{(c, V)}>0$ and $D(b, V)<$ $D(c, V)$

$\Rightarrow D_{(a, V)}>0$ and $D_{(c, V)}>0$ and $D(a, V)<D(c, V)$

(b) $D_{(b, V)}>0$ and $D_{(c, V)}>0$ and $D(b, V)=$ $D(c, V)$ and $D(b, m) \leq D(c, m)$

$\Rightarrow D_{(a, V)}>0$ and $D_{(c, V)}>0$ and

$D(a, V)=D(c, V)$ and

$D(a, m)<D(c, m)$
$\Rightarrow a<_{R G B} c$.

2.2.3 $\left(D_{(a, V)}<0\right.$ and $D_{(b, V)}<0$ and $D(a, V)=$ $D(b, V)$ and $D(a, m)>D(b, m))$ and $b<_{R G B}$ $c$.

From the definition of $<_{R G B}$ would follow that:

(a) $D_{(b, V)}<0$ and $D_{(c, V)} \geq 0$

$\Rightarrow D_{(a, V)}<0$ and $D_{(c, V)} \geq 0$

(b) $D_{(b, V)}<0$ and $D_{(c, V)}<0$ and $D(b, V)>$ $D(c, V)$

$\Rightarrow D_{(a, V)}<0$ and $D_{(c, V)}<0$ and $D(a, V)>D(c, V)$

(c) $D_{(b, V)}<0$ and $D_{(c, V)}<0$ and $D(b, V)=$ $D(c, V)$ and $D(b, m) \geq D(c, m)$

$\Rightarrow D_{(a, V)}<0$ and $D_{(c, V)}<0$ and

$D(a, V)=D(c, V)$ and

$D(a, m)>D(c, m)$

$\Rightarrow a<_{R G B} c$.

$2.3\left(D_{(a, V)}=D_{(b, V)} \neq 0\right.$ and $D(a, m)=D(b, m)$ and $\left.\theta_{a}^{\prime}<_{\theta} \theta_{b}^{\prime}\right)$ and $b<_{R G B} c$.

From the definition of $<_{R G B}$ would follow that:

(a) $D_{(a, V)}=D_{(b, V)}<0$

1. $D_{(c, V)} \geq 0$

2. $D_{(c, V)}<0$ and $D(b, V)>D(c, V)$

3. $D_{(c, V)}<0$ and $D(b, V)=$ $D(c, V)$ and $D(b, m)>D(c, m)$

4. $D_{(b, V)}=D_{(c, V)}$ and $D(b, m)=$ $D(c, m)$ and $\theta_{b}^{\prime}<{ }_{\theta} \theta_{c}^{\prime}$

(b) $D_{(a, V)}=D_{(b, V)}>0$

1. $D_{(c, V)}>0$ and $D(b, V)<D(c, V)$

2. $D_{(c, V)}>0$ and $D(b, V)=$ $D(c, V)$ and $D(b, m)<D(c, m)$

3. $D_{(b, V)}=D_{(c, V)}$ and $D(b, m)=$ $D(c, m)$ and $\theta_{b}^{\prime}<_{\theta} \theta_{c}^{\prime}$

$\Rightarrow a<_{R G B} c$

2.4.1 $\left(D_{(a, V)}=D_{(b, V)}=0\right.$ and $D(a, m) \cos \left(\theta_{a}^{\prime}\right)<$ $\left.D(b, m) \cos \left(\theta_{b}^{\prime}\right)\right)$ and $b<_{R G B} c$

From the definition of $<_{R G B}$ would follow:

(a) $D_{(c, V)}>0$ 
(b) $D_{(b, V)}=D_{(c, V)}$ and $D(b, m) \cos \left(\theta_{b}^{\prime}\right) \leq D(c, m) \cos \left(\theta_{c}^{\prime}\right)$

$\Rightarrow a<_{R G B} c$

2.4.3 $\left(D_{(a, V)}=D_{(b, V)}=0\right.$ and $D(a, m) \cos \left(\theta_{a}^{\prime}\right)=$ $D(b, m) \cos \left(\theta_{b}^{\prime}\right)$ and $D(a, m) \sin \left(\theta_{a}^{\prime}\right)<D(b, m)$ $\left.\sin \left(\theta_{b}^{\prime}\right)\right)$ and $b<_{R G B} c$

From the definition of $<_{R G B}$ would follow:

(a) $D_{(c, V)}>0$

(b) $D_{(b, V)}=D_{(c, V)}$ and $D(b, m) \cos \left(\theta_{b}^{\prime}\right)<D(c, m) \cos \left(\theta_{c}^{\prime}\right)$

(c) $D_{(b, V)}=D_{(c, V)}$ and $D(b, m) \cos \left(\theta_{b}^{\prime}\right)=D(c, m) \cos \left(\theta_{c}^{\prime}\right)$ and $D(b, m) \sin \left(\theta_{b}^{\prime}\right)<D(c, m) \sin \left(\theta_{c}^{\prime}\right)$

$\Rightarrow a \leq_{R G B} c$.

We now prove that our new ordering $\leq_{R G B}$ in RGB is compatible with the complement $c o$ : for all colours c, c' in RGB it holds that

$$
c \leq_{R G B} c^{\prime} \Leftrightarrow c o(c) \geq_{R G B} c o\left(c^{\prime}\right) .
$$

\section{Proof}

$1.1\left(D_{(c, V)}<0\right.$ and $\left.D_{\left(c^{\prime}, V\right)}>0\right)$

$$
\begin{aligned}
D_{(c, V)}<0 & \Leftrightarrow r_{c}+g_{c}+b_{c}<3 / 2 \\
& \Leftrightarrow 3-\left(r_{c}+g_{c}+b_{c}\right)>3-3 / 2 \\
& \Leftrightarrow\left(1-r_{c}\right)+\left(1-g_{c}\right)+\left(1-b_{c}\right) \\
& \Leftrightarrow 3 / 2 \\
& \Leftrightarrow D_{(c o(c), V)}>0
\end{aligned}
$$

So we get $\left(D_{(c, V)}<0\right.$ and $\left.D_{\left(c^{\prime}, V\right)}>0\right) \Leftrightarrow$ $\left(D_{(c o(c), V)}>0\right.$ and $\left.D_{\left(c o\left(c^{\prime}\right), V\right)}<0\right)$.

$1.2\left(D_{(c, V)}=0\right.$ and $\left.D_{\left(c^{\prime}, V\right)}>0\right) \Leftrightarrow\left(D_{(c o(c), V)}=\right.$ 0 and $\left.D_{\left(c o\left(c^{\prime}\right), V\right)}<0\right)$.

$1.3\left(D_{(c, V)}<0\right.$ and $\left.D_{\left(c^{\prime}, V\right)}=0\right) \Leftrightarrow\left(D_{(c o(c), V)}>\right.$ 0 and $\left.D_{\left(c o\left(c^{\prime}\right), V\right)}=0\right)$.

2.1.1 $\left(D_{(c, V)}>0 \quad\right.$ and $\quad D_{\left(c^{\prime}, V\right)}>$ 0 ) and $D(c, V)<D\left(c^{\prime}, V\right)$, where

$$
\begin{aligned}
D(c, V) & =\frac{\left|r_{c}+g_{c}+b_{c}-3 / 2\right|}{\left.\sqrt{3}+b_{c}\right)+3 / 2 \mid} \\
& \left.=\frac{\mid-\left(r_{c}+g_{c}+\sqrt{3}\right.}{\sqrt{3}}\right) \\
& =\frac{\left|3-\left(r_{c}+g_{c}+b_{c}\right)-3 / 2\right|}{\sqrt{3}} \\
& =\frac{\left|\left(1-r_{c}\right)+\left(1-g_{c}\right)+\left(1-b_{c}\right)-3 / 2\right|}{\sqrt{3}} \\
& =D(c o(c), V) .
\end{aligned}
$$

So we get $\left(D_{(c, V)}>0\right.$ and $\left.D_{\left(c^{\prime}, V\right)}>0\right)$ and $D(c, V)<D\left(c^{\prime}, V\right) \Leftrightarrow\left(D_{(c o(c), V)}<0\right.$ and $\left.D_{\left(c o\left(c^{\prime}\right), V\right)}<0\right)$ and $D(\operatorname{co}(c), V)<$ $D\left(\operatorname{co}\left(c^{\prime}\right), V\right)$.

2.2.1 $\left(D_{(c, V)}<0 \quad\right.$ and $D_{\left(c^{\prime}, V\right)}<$ $0)$ and $D(c, V)>D\left(c^{\prime}, V\right) \Leftrightarrow\left(D_{(c o(c), V)}>\right.$ 0 and $\left.D_{\left(c o\left(c^{\prime}\right), V\right)}>0\right)$ and $D(c o(c), V)>$ $D\left(c o\left(c^{\prime}\right), V\right)$.

2.1.3 $\left(D_{(c, V)}>0\right.$ and $D_{\left(c^{\prime}, V\right)}>0$ and $D(c, V)=$ $\left.D\left(c^{\prime}, V\right)\right)$ and $D(c, m)<D\left(c^{\prime}, m\right)$, where

$D(c, m)<D\left(c^{\prime}, m\right)$

$\Leftrightarrow\left(r_{c}-1 / 2\right)^{2}+\left(g_{c}-1 / 2\right)^{2}+\left(b_{c}-1 / 2\right)^{2}<$ $\left(r_{c^{\prime}}-1 / 2\right)^{2}+\left(g_{c^{\prime}}-1 / 2\right)^{2}+\left(b_{c^{\prime}}-1 / 2\right)^{2}$

$\Leftrightarrow r_{c}^{2}-r_{c}+g_{c}^{2}-g_{c}+b_{c}^{2}-b_{c}<r_{c^{\prime}}^{2}-r_{c^{\prime}}+g_{c^{\prime}}^{2}-$ $g_{c^{\prime}}+b_{c^{\prime}}^{2}-b_{c^{\prime}}$

$\Leftrightarrow 1-2 r_{c}+r_{c}^{2}-1+r_{c}+1-2 g_{c}+g_{c}^{2}-1+g_{c}+$ $1-2 b_{c}+b_{c}^{2}-1+b_{c}<1-2 r_{c^{\prime}}+r_{c^{\prime}}^{2}-1+r_{c^{\prime}}+$ $1-2 g_{c^{\prime}}+g_{c^{\prime}}^{2}-1+g_{c^{\prime}}+1-2 b_{c^{\prime}}+b_{c^{\prime}}^{2}-1+b_{c^{\prime}}$ $\Leftrightarrow\left(1-r_{c}\right)^{2}+1 / 4-\left(1-r_{c}\right)+\left(1-g_{c}\right)^{2}+$ $1 / 4-\left(1-g_{c}\right)+\left(1-b_{c}\right)^{2}+1 / 4-\left(1-b_{c}\right)<$ $\left(1-r_{c^{\prime}}\right)^{2}+1 / 4-\left(1-r_{c^{\prime}}\right)+\left(1-g_{c^{\prime}}\right)^{2}+1 / 4-$ $\left(1-g_{c^{\prime}}\right)+\left(1-b_{c^{\prime}}\right)^{2}+1 / 4-\left(1-b_{c^{\prime}}\right)$

$\Leftrightarrow\left(\left(1-r_{c}\right)-1 / 2\right)^{2}+\left(\left(1-g_{c}\right)-1 / 2\right)^{2}+((1-$ $\left.\left.\left.b_{c}\right)-1 / 2\right)^{2}\right)<\left(\left(1-r_{c^{\prime}}\right)-1 / 2\right)^{2}+\left(\left(1-g_{c^{\prime}}\right)-\right.$ $\left.1 / 2)^{2}+\left(\left(1-b_{c^{\prime}}\right)-1 / 2\right)^{2}\right)$

$\Leftrightarrow D(c o(c), m)<D\left(c o\left(c^{\prime}\right), m\right)$.

So we get $\left(D_{(c, V)}>0\right.$ and $\left.D_{\left(c^{\prime}, V\right)}>0\right)$ and $D(c, V)=D\left(c^{\prime}, V\right)$ and $D(c, m)<D\left(c^{\prime}, m\right) \Leftrightarrow$

$\left(D_{(c o(c), V)}<0\right.$ and $\left.D_{\left(c o\left(c^{\prime}\right), V\right)}<0\right)$ and

$D(c o(c), V)=D\left(c o\left(c^{\prime}\right), V\right)$ and $D(c o(c), m)<$ $D\left(c o\left(c^{\prime}\right), m\right)$.

2.2.3 $\left(D_{(c, V)}<0\right.$ and $\left.D_{\left(c^{\prime}, V\right)}<0\right)$ and $D(c, V)=$ $D\left(c^{\prime}, V\right)$ and $D(c, m) \quad>\quad D\left(c^{\prime}, m\right) \Leftrightarrow$ $\left(D_{(c o(c), V)}>0\right.$ and $D_{\left(c o\left(c^{\prime}\right), V\right)}>$ $0)$ and $D(c o(c), V)=$

$D\left(c o\left(c^{\prime}\right), V\right)$ and $D(c o(c), m)>D\left(c o\left(c^{\prime}\right), m\right)$.

$2.3 D_{(c, V)}=D_{\left(c^{\prime}, V\right)} \neq 0$ and $D(c, m)=D\left(c^{\prime}, m\right)$

A. $\theta_{c}^{\prime} \in[0, \pi[$

i. $\theta_{c^{\prime}}^{\prime} \in[0, \pi[$

From $c \leq_{R G B} c^{\prime}$ it follows that $\theta_{c}^{\prime} \leq$ $\theta_{c^{\prime}}^{\prime}$. By definition of co we get: $\theta_{c o(c)}^{\prime}=\theta_{c}^{\prime}+\pi$ and $\theta_{c o\left(c^{\prime}\right)}^{\prime}=\theta_{c^{\prime}}^{\prime}+\pi$ and hence from $\theta_{c}^{\prime} \leq \theta_{c^{\prime}}^{\prime}$ we get $\theta_{c}^{\prime}+\pi \leq$ $\theta_{c^{\prime}}^{\prime}+\pi$, i.e., $\theta_{c o(c)}^{\prime} \leq \theta_{c o\left(c^{\prime}\right)}^{\prime}$. 
ii. $\theta_{c^{\prime}}^{\prime} \in[\pi, 2 \pi[$

From $c \leq_{R G B} c^{\prime}$ it follows that $\theta_{c}^{\prime} \leq$ $\theta_{c^{\prime}}^{\prime}$. By definition of co we get: $\theta_{c o(c)}^{\prime}=\theta_{c}^{\prime}+\pi$ and $\theta_{c o\left(c^{\prime}\right)}^{\prime}=\theta_{c^{\prime}}^{\prime}-\pi$ and hence from $\theta_{c}^{\prime}+\pi \in[\pi, 2 \pi[$ and $\theta_{c^{\prime}}^{\prime}-\pi \in\left[0, \pi\left[\right.\right.$ we get $\theta_{c o\left(c^{\prime}\right)}^{\prime} \leq \theta_{c o(c)}^{\prime}$.

B. $\theta_{c}^{\prime} \in[\pi, 2 \pi[$

i. $\theta_{c^{\prime}}^{\prime} \in[0, \pi[$, impossible

ii. $\theta_{c^{\prime}}^{\prime} \in[\pi, 2 \pi[$

From $c \leq_{R G B} c^{\prime}$ it follows that $\theta_{c}^{\prime} \geq$ $\theta_{c^{\prime}}^{\prime}$. By definition of $c o$ we get: $\theta_{c o(c)}^{\prime}=\theta_{c}^{\prime}-\pi$ and $\theta_{c o\left(c^{\prime}\right)}^{\prime}=\theta_{c^{\prime}}^{\prime}-\pi$ and hence from $\theta_{c}^{\prime} \geq \theta_{c^{\prime}}^{\prime}$ we get $\theta_{c}^{\prime}-\pi \geq$ $\theta_{c^{\prime}}^{\prime}-\pi$, i.e., $\theta_{c o(c)}^{\prime} \geq \theta_{c o\left(c^{\prime}\right)}^{\prime}$.

2.4.1 $D_{(c, V)}=D_{\left(c^{\prime}, V\right)}=0$ and $D(c, m) \cos \left(\theta_{c}^{\prime}\right)<$ $D\left(c^{\prime}, m\right) \cos \left(\theta_{c^{\prime}}^{\prime}\right)$, where

$D(c, m) \cos \left(\theta_{c}^{\prime}\right)<D\left(c^{\prime}, m\right) \cos \left(\theta_{c^{\prime}}^{\prime}\right)$

$\Leftrightarrow-D(c, m) \cos \left(\theta_{c}^{\prime}\right)>-D\left(c^{\prime}, m\right) \cos \left(\theta_{c^{\prime}}^{\prime}\right)$

$\Leftrightarrow D(c, m) \cos \left(\left(\theta_{c}^{\prime}+\pi\right) \bmod 2 \pi\right)>$

$D\left(c^{\prime}, m\right) \cos \left(\left(\theta_{c^{\prime}}^{\prime}+\pi\right) \bmod 2 \pi\right)$

$\Leftrightarrow D(c, m) \cos \left(\theta_{c o(c)}^{\prime}\right)>D\left(c^{\prime}, m\right) \cos \left(\theta_{c o\left(c^{\prime}\right)}^{\prime}\right)$,

where we have used the property that

$$
\cos (x+\pi)=\cos (x-\pi)=-\cos (x)
$$

and

$$
(x+\pi) \bmod 2 \pi=x-\pi \text { or } x+\pi
$$

for all $x \in[0,2 \pi]$.

So we get $D_{(c, V)}=D_{\left(c^{\prime}, V\right)}=0$ and

$D(c, m) \cos \left(\theta_{c}^{\prime}\right)<D\left(c^{\prime}, m\right) \cos \left(\theta_{c^{\prime}}^{\prime}\right) \Leftrightarrow$

$D_{(c o(c), V)}=D_{\left(\operatorname{co}\left(c^{\prime}\right), V\right)}=0$ and

$D(c, m) \cos \left(\theta_{c o(c)}^{\prime}\right)>D\left(c^{\prime}, m\right) \cos \left(\theta_{c o\left(c^{\prime}\right)}^{\prime}\right)$.

2.4.3 $D_{(c, V)}=D_{\left(c^{\prime}, V\right)}=0$ and $D(c, m) \cos \left(\theta_{c}^{\prime}\right)=$ $D\left(c^{\prime}, m\right) \cos \left(\theta_{c^{\prime}}^{\prime}\right)$ and $D(c, m) \sin \left(\theta_{c}^{\prime}\right)<$

$D\left(c^{\prime}, m\right) \sin \left(\theta_{c^{\prime}}^{\prime}\right)$, where

$D(c, m) \cos \left(\theta_{c}^{\prime}\right)=D\left(c^{\prime}, m\right) \cos \left(\theta_{c^{\prime}}^{\prime}\right)$

$\Leftrightarrow-D(c, m) \cos \left(\theta_{c}^{\prime}\right)=-D\left(c^{\prime}, m\right) \cos \left(\theta_{c^{\prime}}^{\prime}\right)$

$\Leftrightarrow D(c, m) \cos \left(\left(\theta_{c}^{\prime}+\pi\right) \bmod 2 \pi\right)=$

$D\left(c^{\prime}, m\right) \cos \left(\left(\theta_{c^{\prime}}^{\prime}+\pi\right) \bmod 2 \pi\right)$

$\Leftrightarrow D(c, m) \cos \left(\theta_{c o(c)}^{\prime}\right)=D\left(c^{\prime}, m\right) \cos \left(\theta_{c o\left(c^{\prime}\right)}^{\prime}\right)$

and

$D(c, m) \sin \left(\theta_{c}^{\prime}\right)<D\left(c^{\prime}, m\right) \sin \left(\theta_{c^{\prime}}^{\prime}\right)$
$\Leftrightarrow-D(c, m) \sin \left(\theta_{c}^{\prime}\right)>-D\left(c^{\prime}, m\right) \sin \left(\theta_{c^{\prime}}^{\prime}\right)$

$\Leftrightarrow D(c, m) \sin \left(\left(\theta_{c}^{\prime}+\pi\right) \bmod 2 \pi\right)>$

$D\left(c^{\prime}, m\right) \sin \left(\left(\theta_{c^{\prime}}^{\prime}+\pi\right) \bmod 2 \pi\right)$

$\Leftrightarrow D(c, m) \sin \left(\theta_{c o(c)}^{\prime}\right)>D\left(c^{\prime}, m\right) \sin \left(\theta_{c o\left(c^{\prime}\right)}^{\prime}\right)$,

where we have used the property that

$$
\sin (x+\pi)=\sin (x-\pi)=-\sin (x)
$$

and

$$
(x+\pi) \bmod 2 \pi=x-\pi \text { or } x+\pi
$$

for all $x \in[0,2 \pi]$.

So we get $D_{(c, V)}=D_{\left(c^{\prime}, V\right)}=0$ and

$D(c, m) \cos \left(\theta_{c}^{\prime}\right)=D\left(c^{\prime}, m\right) \cos \left(\theta_{c^{\prime}}^{\prime}\right)$ and

$D(c, m) \sin \left(\theta_{c}^{\prime}\right)<D\left(c^{\prime}, m\right) \sin \left(\theta_{c^{\prime}}^{\prime}\right) \Leftrightarrow$

$D_{(c o(c), V)}=D_{\left(c o\left(c^{\prime}\right), V\right)}=0$ and

$D(c, m) \cos \left(\theta_{c o(c)}^{\prime}\right)=D\left(c^{\prime}, m\right) \cos \left(\theta_{c o\left(c^{\prime}\right)}^{\prime}\right)$ and

$D(c, m) \sin \left(\theta_{c o(c)}^{\prime}\right)>D\left(c^{\prime}, m\right) \sin \left(\theta_{c o\left(c^{\prime}\right)}^{\prime}\right)$.

$\Rightarrow c \leq_{R G B} c^{\prime} \stackrel{!}{\Leftrightarrow} c o(c) \geq_{R G B} c o\left(c^{\prime}\right)$.

\section{Acknowledgments}

This research was financially supported by the GOAproject 12.0515 .03 of Ghent University.

\section{References}

1. V. De Witte, "Colour Morphology with Application to Image Magnification," PhD Thesis, Ghent University, Belguim (2007).

2. College of Saint Benedict - Saint John's University, "Color depth and color spaces," http://www.csbsju.edu/itservices/teaching/ c_space/colors.htm.

3. L. J. Sartor and A. R. Weeks, "Morphological operations on color images," Journal of Electronic Imaging, 10, 2, 548-559 (2001).

4. J. Li and Y. Li, "Multivariate mathematical morphology based on principal component analysis: initial results in building extraction," International Archives for Photogrammetry, Remote Sensing and Spatial Information Sciences, 35, B7, 1168-1173 (2004).

5. Francisco Flórez-Revuelta, "Ordering of the RGB space with a growing self-organizing network. Application to color mathematical morphology," Lecture Notes in Computer Science, 3696, 385-390 (2005).

6. M. L. Comer and E. J. Delp, "Morphological operations for color image processing," Journal of Electronic Imaging, 8, 3, 279-289 (1999). 
7. J. Angulo and J. Serra, "Morphological coding of color images by vector connected filters" IEEE Proceedings of IEEE rth International Symposium on Signal Processing and Its Applications ISSPA 2003, 33, 69-72 (2003).
8. V. De Witte and S. Schulte and E. E. Kerre and A. Ledda and W. Philips, "Morphological image interpolation to magnify images with sharp edges," Lecture Notes in Computer Science, 4141, 381-393 (2006). 